\title{
Política y ciudadanía: el liderazgo de María Guadalupe Urzúa en la Confederación Nacional Campesina, 1953-1957
}

\author{
Politics and Citizenship: Maria Guadalupe Urzúa's Leadership \\ of the Confederacion Nacional Campesina, 1953-1957
}

María Teresa Fernández Aceves

CIESAS-OCCIDENTE, mfernandez@ciesas.edu.mx

Este artículo examina cómo María Guadalupe Urzúa Flores buscó consolidar su liderazgo y su ascenso político en la Confederación Nacional Campesina (CNC) y el Partido Revolucionario Institucional (PRI) por medio de la dirigencia de la Secretaría de Acción Femenil de la CNC; explora cómo fue el proceso de afiliación de las mujeres al PRI en la década de 1950 e identifica las políticas sociales que se trataron de instrumentar desde la Secretaría de Acción Femenil entre 1953 y 1955, dos años claves en la participación política de las mujeres en México.

PalabRas ClaVE: políticas sociales, sufragio femenino, elecciones, mujeres, nutrición.

This article examines how María Guadalupe Urzúa Flores worked to consolidate her leadership and political ascent in Mexico's Confederación Nacional Campesina (CNC, "National Confederation of Peasants"), and in the Partido Revolucionario Institucional (PRI, "Institutional Revolutionary Party") during her time as Director of the cNc's Secretariat of Women's Action. It explores the process through which women became affiliated to the PRI In the decade of 1950, and identifies the social policies that the Secretariat of Women's Action strove to have implemented from 1953 to 1955; two key years for the political participation of women in Mexico.

KEYWORDS: social policies, women's suffrage, elections, women, nutrition.

Fecha de recepción del artículo: 13 de mayo de 2016 / Fecha de aprobación: 10 de noviembre de 2016 / Fecha de recepción de la versión final: 7 de diciembre de 2016

n diciembre de 1954, catorce representantes de las ligas fe-
meniles del Comité Regional de la Confederación Nacional
Campesina (CNC) del municipio Francisco I. Madero,
Coahuila, le manifestaron una preocupación a María Guadalupe
Urzúa Flores, secretaria de Acción Femenil de la CNC. De acuerdo 
con las firmantes, al crearse los Centros Pilotos de Bienestar Social de la Secretaría de Salubridad y Asistencia (ssa) en la región de la Laguna, empezó a decaer la moral de las ligas femeniles porque los médicos de estos Centros fomentaban la división entre las campesinas. Los médicos contaban con todo el respaldo y facilidades para realizar su trabajo; y pretendían que las ligas femeniles dependieran sólo de dichos Centros. Para los médicos, los Centros de cada ejido debían ser la casa de la mujer campesina, por tanto, sugerían que ellas sólo acudieran a estos lugares. Las representantes de las ligas femeniles se quejaban de que las aportaciones de los campesinos para los servicios médicos, se utilizaban en la construcción de esos Centros, en vez de atender la salud de las familias campesinas. Ellas destacaban que el general Lázaro Cárdenas había dotado con derechos agrarios a su comunidad, pero "en el transcurso de los años no se [les habían] dado las garantías económicas y sociales para formar un hogar modesto y forjar una vida de honestidad para nuestras propias familias". ${ }^{1}$ Le pedían a Urzúa Flores les informara “el camino a seguir pero en una forma de veracidad que pueda simentar [sic] no solamente las esperanzas sino la realidad". ${ }^{2}$ Ellas buscaban persuadirla para que como líder campesina realizara acciones que contribuyeran a mejorar su calidad de vida.

Esta queja y consulta de las catorce dirigentes de las ligas campesinas de la región de la Laguna nos introducen a varios procesos y políticas que estaban en competencia cuando Urzúa Flores presidía la Secretaría de Acción Femenil de la CNC (1953-1957). Ella comenzó su trabajo político desde abajo, los rangos inferiores locales de la liga femenil del Comité Agrario y en la sección femenil del Partido de la Revolución Mexicana (PRM) y del Partido Revolucionario Institucional (PRI) en San Martín Hidalgo, Jalisco. A partir de esta experiencia política entre las décadas de 1930 y 1950, a Urzúa Flores le quedaba muy claro que era necesario detallar aún más las acciones de las ligas y acercarse a ellas. Reconocía que estas mujeres,

${ }^{1}$ Colección Documental María Guadalupe Urzúa Flores-Biblioteca Carmen Castañeda García del Ciesas-Occidente (en adelante CDMGUf-BCCG-CO), 2. Acción Política, 2.1 Acción Femenina-CNC, caja 19, exp. 625, carta diciembre de 1954, ff. 4.

${ }^{2}$ doc. cit. 
como base política, eran foco de disputa de diferentes grupos políticos porque contribuían a consolidar el cimiento de organizaciones e intereses políticos. Asimismo en repetidos casos se dio un fuerte impulso político a determinados líderes que de distintas maneras manipulaban a esas bases femeniles.

En este periodo el ejido y la reforma agraria ya habían perdido la preponderancia que tuvieron en el decenio de 1930; se daba paso a una política agraria que favorecía la modernización de la agricultura comercial, la cual generó marcadas desigualdades. ${ }^{3}$ Sin embargo, los procesos y políticas estatales cambiantes estaban generando diversas pugnas entre el personal por la asignación y distribución de recursos. Por una parte, en la región de la Laguna los médicos de la SsA y las ligas femeniles de la CNC se disputaban quién debía liderar a las campesinas. ${ }^{4}$ Por otra parte, después de la creación del PRI en 1946 y del reconocimiento del sufragio femenino en 1953, las funciones de las secretarias femeniles se ampliaron para incrementar el número de afiliadas, pero de una manera controlada y disciplinada. ${ }^{5} \mathrm{Al}$ llevarlo a cabo, hubo roces y resistencias con otros grupos políticos, como lo ilustra muy bien el caso del liderazgo de Urzúa Flores en la Secretaría de Acción Femenina de la CNC. Por último, diferentes secretarías de Estado pretendían atender algunos de los diversos problemas (pobreza, salud, nutrición y educación) que aquejaban al México del decenio de 1950 por medio de políticas sociales, que algunas veces concordaban, en otras se coordinaban y en otras entraban en fuerte competencia entre sí, pero al final buscaban la mo-

${ }^{3}$ Cynthia Hewitt de Alcántara, La modernización de la agricultura mexicana, 1940 1970 (México: Siglo XXI Editores, 1988), 99.

${ }^{4}$ Para un excelente análisis sobre la construcción y puesta en marcha de una política radical de higiene y salud rural, primero en Michoacán y después a nivel federal, elaborada por los médicos nicolaitas, el Dr. Jesús Díaz Barriga (1891-1971) y el Dr. Enrique Arreguín Vélez (1907-1989), veáse Ana María Kapelusz-Poppi, "Physician Activists and the Development of Rural Health in Postrevolutionary Mexico", Radical History Revire (80) (primavera 2001): 35-50; Ana María Kapelusz-Poppi, "Rural Health and Statu Construcción in Post-Revolucionar Mexico: The Nicolita Project foro Rural Medical Cervices", The Américas 58(2) (octubre 2001): 261-283.

${ }^{5}$ Gisela Zaremberg, Mujeres, votos y asistencia social en el México priista y la Argentina peronista (México: FlaCso México, 2009), 290. 
dernización de prácticas educativas, de salud, de alimentación y de trabajo para impulsar un desarrollo económico. ${ }^{6}$

Desde las perspectivas del discurso de la CNC y de las mujeres, en este artículo examino cómo Urzúa Flores buscó consolidar su liderazgo y su ascenso político por medio de la dirigencia de la Secretaría de Acción Femenil de la CNC y exploro cómo fue el proceso de afiliación de las mujeres al PRI desde la Secretaría de Acción Femenil entre 1953 y 1955, dos años claves en la participación política de las mujeres en México: el reconocimiento del sufragio femenino (1953), las campañas electorales de mujeres para diputaciones federales en 1955 y el ejercicio del voto de las mujeres en esta contienda. En este artículo avanzo en mi conceptualización de Urzúa Flores como una gestora, para matizar mi visión benévola de gestora como alguien que sólo busca el bienestar, a una en el que el gestor o gestora demanda el bienestar, pero con un interés de grupo, ya sea de un partido político, organización o grupo político. ${ }^{7}$ Me baso princi-

${ }^{6}$ Para una discusión sobre estos temas véase Mary Kay Vaughan, "Modernizing Patriarchy: State Policies, Rural Households, and Women in Mexico, 1930-1940", en Hidden Histories of Gender and State in Latin America, ed. Maxine Molyneux y Elizabeth Dore, 194-214 (Durham: Duke University Press, 2000); Mary Kay Vaughan, La politica cultural de la revolución (México: Fondo de Cultura Económica, 2001); Oresta López, Alfabeto y enseñanzas domésticas. El arte de ser maestra rural en el Valle del Mezquital (México: Ciesas, Consejo Estatal para la Cultura y las Artes de Hidalgo, 2001); Patience A. Schell, Church and State Education in Revolutionary Mexico City (Tucson: University of Arizona Press, 2003); Jocelyn Olcott, Revolutionary Women in Postrevolutionary Mexico (Durham: Duke University Press, 2005); Sandra Aguilar-Rodríguez, "Cooking Modernity: Nutrition Policies, Class, and Gender in 1940s and 1950s Mexico City”, The Americas, 64(2) (octubre 2007): 177-205; Susie S. Porter, Mujeres y trabajo en la ciudad de México: condiciones materiales y discursos públicos (1879-1931) (Zamora: El Colegio de Michoacán, 2008); Ann S. Blum, Domestic Economies: Family, Work, and Welfare in Mexico City, 1884-1943 (Lincoln: University Nebraska Press, 2009); Alicia Civera Cerecero Cerecedo, "El cooperativismo en la escuela rural del México de los años treinta / The Cooperativism in the Mexican Rural School, 1930s", Anuario de Estudios Americanos 67(2) (julio-diciembre de 2010): 467-491; Nichole Sanders, Gender and Welfare in Mexico: the Consolidation of a Postrevolutionary State (University Park: Pennsylvania State University Press, 2011).

${ }^{7}$ En Mujeres en el cambio social en el siglo XX mexicano sostengo que "por gestora entiendo que se denomina a aquella persona que promueve y lucha por la solución de problemas locales específicos que buscan beneficiar a las comunidades; pero no necesariamente pertenece a la localidad. Su compromiso con el servicio social y con la solución de problemas contribuye y ejerce influencia en la formación o ejecución de la política 
palmente en documentación inédita de la Colección Documental María Guadalupe Urzúa Flores localizada en la Biblioteca Carmen Castañeda García del Ciesas-Occidente, la complemento con documentos de otros acervos documentales.

Este artículo se enfoca en la movilidad política de Urzúa Flores dentro de las estructuras corporativas del PRI y contribuye a la propuesta de la historiadora Mary Kay Vaughan, quien sostiene que es necesario deconstruir al Estado después de la década de 1940 para desmenuzar cómo opera en las escalas local, regional y nacional. ${ }^{8}$ También aporta a las historiografías sobre la participación de las mujeres en la política formal y en los estudios históricos sobre políticas sociales. ${ }^{9}$ Primero, describo el rápido ascenso de Urzúa Flores que pasó de ser regidora y vicepresidenta del municipio de San Martín Hidalgo, Jalisco (1951-1953), a dirigir la Secretaría de Acción Femenil de la CNC a nivel federal a partir del 20 de agosto de 1953. Segundo, analizo las tácticas y estrategias que usó Urzúa Flores para construir su liderazgo dentro de la CNC y hacer más visibles a las ligas femeniles en las comunidades agrarias, en la vida cotidiana y en la política corporativa y autoritaria del PRI y de la CNC. Tercero, profundizo en los Cursos de Capacitación Intensivos para Mujeres Campesinas organizados por Urzúa Flores desde la CNC y el vínculo

pública; además, puede o no tener una afiliación institucional a un sindicato, partido político u organización social o política. Su papel es identificar las necesidades locales y convertirse en intermediario cultural o político entre distintas instituciones y fuerzas culturales, políticas y sociales. Conoce bien la estructura de la burocracia estatal e identifica actores políticos influyentes y tomadores de decisiones, lo cual será clave para persuadirlos o disuadirlos". María Teresa Fernández Aceves, Mujeres en el cambio social en el siglo XX mexicano (México: Siglo XXI Editores, Ciesas, 2014), 274-275.

${ }^{8}$ Los siguientes estudios históricos se han adentrado en revisar y en el diseño y la instrumentación de políticas sociales, véase Mary Kay Vaughan, "Cultural Approaches to Peasant Politics in the Mexican Revolution", The Hispanic American Historical Review 79(2) (mayo 1999): 299. Para una discusión sobre la diversidad de facetas del Estado como "actor" y la pluralidad de los actores sociales desde una perspectiva descentrada véase Elsie Rockwell y Eugenia Roldán Vera, "State Governance and Civil Society in Education: Revisiting the Relationship", Paedagogica Historica 49(1) (2013): 1-16.

${ }^{9}$ Vaughan, "Modernizing Patriarchy"; Aguilar-Rodríguez, "Cooking Modernity"; Sanders, Gender and Welfare in Mexico; Gabriela Castañeda López y Ana Cecilia Rodríguez de Romo, Desafiando a la tradición: las primeras egresadas de las escuelas de medicina de México, 1887-1936 (México: Academia Nacional de Medicina de México, 2014). 
entre la Secretaría de Educación Pública y la Junta Nacional para el Mejoramiento de la Alimentación del Instituto Nacional de Nutrición para poner en marcha políticas educativas y de nutrición dirigidas a mujeres. Los hacedores de estas políticas públicas percibían principalmente a las mujeres como amas de casa y madres, quienes debían aprender sobre la ciencia doméstica. Con este entrenamiento, las campesinas podían convertirse en agentes de modernización, leales priistas, pero conocedoras de sus derechos y deberes cívicos, políticos y sociales. Concluyo que las gestiones de Urzúa Flores y las campañas de orientación cívica y política rindieron productos inmediatos al incrementarse el número de afiliadas y formar a nuevos cuadros de dirigentes medios, pero aún con muchos sinsabores en la participación de la política formal por la competencia desigual entre hombres y mujeres. La documentación inédita en la Colección Documental María Guadalupe Urzúa Flores (CDMGUf) corrobora el argumento de Maxine Molyneux de que la igualdad jurídica

era una igualdad falsa, en el sentido de que al eliminar las diferencias pertinentes (como la maternidad) se daba por hecho un mismo terreno de juego para ambos sexos. Al tratar a las mujeres como hombres, la simple igualdad pasaba por alto las desigualdades de circunstancia y de oportunidad, y podía tener el efecto perverso de reproducir la desigualdad mediante formas ocultas de discriminación. ${ }^{10}$

\section{De LA POLÍTICA MUNICIPAL A LA POLÍTICA FEDERAL}

Como describo en el libro Mujeres en el cambio social, María Guadalupe Urzúa Flores (1912-2004) provenía de una familia de la clase media rural. Huérfana de madre y criada por sus tías Flores Monroy (maestras y una farmacéutica) en San Martín Hidalgo, Jalisco, Urzúa Flores observó de manera cotidiana en la farmacia de su tía Julia, a las familias de campesinos con lepra que llegaban por medicamen-

${ }^{10}$ Maxine Molyneux, "Prefacio", en Los códigos del género. Prácticas del derecho en el México contemporáneo, ed. Helga Baitenmann, Victoria Chenaut y Ann Varley, 11 (México: UNAM-PUEG, Fondo de Desarrollo de las Naciones Unidas para la Mujer, UnifEM, 2010). 
to. La condición de salud de los campesinos, aunada al violento proceso agrario que se dio en este municipio entre los decenios de 1920 y 1930, sensibilizó de manera significativa a Urzúa Flores a favor de los desposeídos.

En la década de 1930, Urzúa Flores se incorporó a las organizaciones agrarias, culturales y deportivas de su pueblo y colaboró con el ayuntamiento para la construcción de una brecha. Entre 1936 y 1937 estuvo a cargo de la liga femenil del Comité Agrario de San Martín Hidalgo, pugnando por las necesidades de viudas y campesinos; y representó a la Secretaría de Acción Femenil del Comité Campesino de Ameca, Jalisco en el PRM (1938). En 1937 fundó en San Martín Hidalgo la academia femenil "Rita Pérez de Moreno" donde se impartían clases de corte, primeros auxilios, tejido de agujas, inglés e historia.

En el siguiente decenio colaboró en ceremonias cívicas, en la fundación de la biblioteca popular y en el centro de alfabetización para campesinos; dirigió la Secretaría de Acción Femenil en el Comité Municipal del PRM-PRI (1942-1946). En este periodo emprendió una de sus obras públicas más importante, la construcción de un hospital en su pueblo. Fue la presidenta del Comité Pro-Hospital de San Martín Hidalgo. Este proyecto la llevó a realizar muchas gestiones en los ámbitos local, regional, nacional y transnacional; realizó rifas, kermeses, corridas de toros y escribió a los sanmartineses en Estados Unidos para que donaran para el hospital. Colaboró con el reconocido dermatólogo jalisciense, el Dr. José Barba Rubio para formar y presidir el Patronato de la Lucha contra la Lepra en su municipio (1947). La dotación de una parcela en la Comunidad Agraria de San Martín Hidalgo (1948), la vinculó más con los problemas campesinos; y fungió como secretaria de la Junta de Mejoramiento Moral, Cívico y Material para trabajar de forma cercana con el ayuntamiento local (1949-1952).

La década de 1950 fue el despunte político de Urzúa Flores gracias al reconocimiento del sufragio femenino en los municipios (1947) y en la federación (1953). Fue secretaria de Acción Femenil en la Liga de Comunidades Agrarias y Sindicatos Campesinos del Estado de Jalisco (1950-1954); encabezó la Secretaría de Salubridad 
y Asuntos Sociales del Comisariado Ejidal de San Martín Hidalgo (1950-1954). En 1951, la Liga de Comunidades Agrarias y Sindicatos Campesinos de Jalisco la comisionó para que se uniera a la campaña presidencial de Adolfo Ruiz Cortines en la entidad. Fue regidora y presidenta electa de San Martín Hidalgo (1951-1953). ${ }^{11}$ En este año participó en diversos congresos y reuniones que debatieron el sufragio femenino y los problemas que enfrentaban las mujeres. El 8 de abril presentó una ponencia sobre los problemas de la mujer ante el Ayuntamiento de San Martín Hidalgo. ${ }^{12}$ Un mes después, el 8 de mayo, en el I Congreso de Ayuntamientos de Jalisco, junto con otras cuatro regidoras, de Guadalajara, Tamazula de Gordiano, Puerto Vallarta y Ahualulco de Mercado, propusieron se atendieran los problemas que aquejaban a las mujeres y se respetaran las leyes para protegerlas. ${ }^{13}$

En julio de 1953, la Liga de Comunidades Agrarias y Sindicatos Campesinos de Jalisco la designó representante del sector campesino en el Congreso Nacional de la Mujer organizado por el PRI. ${ }^{14}$ En este Congreso, Urzúa Flores argumentó que el “otorgamiento de la ciudadanía debe ir aparejado el reconocimiento de sus derechos de dotación de parcela en igualdad con el hombre". ${ }^{15}$ Este planteamiento ya se había propuesto en los congresos de obreras y campesinas de los ańos 1931 y 1933 donde coincidieron los puntos de vista de mujeres comunistas y mujeres del entonces Partido Nacional Revolucionario. Sostuvo que la mujer campesina debía recibir dotación de tierras; consideró necesario que se levantara un censo

${ }^{11}$ Existe una discrepancia sobre las fechas que fue electa regidora y presidenta de San Martín Hidalgo. Los libros de cabildo de esos años no están en el Archivo Municipal de San Martín Hidalgo. Los diferentes curriculum vitae que están en la CDMGUF consignan años distintos. CDMGUF-BCCG-CO, 1. Personal, 1.2 Biografías y curriculum vitae, caja 3, exp. 43-54, 1932-1991.

${ }^{12}$ CDMGuF-BCCG-CO, 2. Acción Política, 2.14.5 Mujeres, caja 143, exp. 5135, Ponencia de María Guadalupe Urzúa Flores regidora de San Martín Hidalgo, 1953, ff. 2.

${ }^{13}$ CDMguf-BCcG-Co, 2. Acción Política, 2.14.5 Mujeres, caja 142, exp. 5107, Ponencia de María Guadalupe Urzúa Flores regidora de San Martín Hidalgo, 1953, ff. 4.

${ }^{14}$ CDMGuf-BCCG-CO, 2. Acción Política, 2.1 Acción Femenina-CNC, caja 19, exp. 589, discurso de Urzúa Flores en el I Congreso de la Mujer, 1953, ff. 9.

${ }^{15}$ CDMGuf-BCCG-CO, 2. Acción Política, 2.1 Acción Femenina-cnc, caja 19, exp. 589 , ponencia, ff. 7 . 
agrario de mujeres; ponderó sobre las dificultades que tendrían las mujeres si obtuvieran parcelas y sostuvo que ellas también tenían derecho a obtener créditos ejidales; criticó las condiciones de violencia en el campo, los latifundios y los cacicazgos. ${ }^{16}$

El 9 agosto de 1953, Urzúa Flores le propuso a Enrique Rodríguez Cano, secretario de la Presidencia de la República, acciones para consolidar las organizaciones de campesinas, expresadas en el I Congreso de la Mujer organizado por el PRI. Como ya lo habían hecho otras líderes campesinas o feministas en las décadas de 1920 y $1930,{ }^{17}$ Urzúa Flores sugería se crearan ligas femeniles en todo el país y fomentar el establecimiento de Juntas de Mejoramiento Moral, Cívico y Material.

Cada uno de los cargos que ostentó Urzúa Flores hasta 1953 fueron parte de la consolidación de un sistema político autoritario, antidemocrático y corporativo en México, en el que el PRI cooptó a sus dirigentes "e inhibió una inclusión masiva de las bases". ${ }^{18}$ Para Gisela Zaremberg, la organización del sector femenil del PRI tuvo una estructura jerárquica, "en la que las máximas dirigentes femeniles eran directamente designadas por el Comité Central Ejecutivo del PRI y las delegadas de los sectores por los comités ejecutivos de

${ }^{16}$ Para un análisis más a fondo de esta propuesta, véase María Teresa Fernández Aceves, "Las políticas de género de la Confederación Nacional Campesina y el liderazgo de María Guadalupe Urzúa Flores, 1950-1960”, en Mexico in Transition: New Persectives on Mexican Agrarian History, Nineteenth and Twentieth Centuries / México y sus transiciones: reconsideraciones sobre la historia agraria mexicana, siglos XIX y XX, ed. Antonio Escobar Ohmstede y Mattew Butler, 565-600 (México: Ciesas, Universidad de Texas-Austin, 2013).

${ }^{17}$ Para una reconstrucción histórica de las ligas de mujeres véase Piedad Peniche Rivero, "El movimiento feminista de Elvia Carrillo Puerto y las igualadas: un liderazgo cultural en Yucatán", en Dos mujeres fuera de serie: Elvia Carillo Puerto y Felipa Poot, ed. Piedad Peniche Rivero y Kathleen R. Martín, 15-69 (Mérida: Instituto de la Cultura de Yucatán, 2007); Stephanie J. Smith, Gender and the Mexican Revolution: Yucatan Women and the Realities of Patriarchy (Chapel Hill: University of North Carolina Press, 2009); Jocelyn Olcott, Revolutionary Women in Postrevolutionary Mexico, Durham, Duke University Press, 2005; “'Take off that streetwalker's dress': Concha Michel and the Cultural Politics of Gender in Postrevolutionary Mexico", Journal of Women's History 21(3) (otoño de 2009): 36-59; Stephanie Mitchell y Patience A. Schell, eds., The Women's Revolution, Mexico (1910-1953) (Nueva York: Roman and Littlefield, 2006).

${ }^{18}$ Zaremberg, Mujeres, votos y asistencia social, 293. 
los mismos" ${ }^{19}$ Urzúa Flores subiría dentro de la estructura del PRI, pero de forma ordenada y subordinada; pasó de los comités municipales, a los estatales, y finalmente, al comité central de la CNC.

\section{LIDERAZGO EN LA CNC}

Urzúa Flores asumió la dirección nacional de la Secretaría de Acción Femenil el 20 de agosto de 1953, trazó un plan de trabajo para la Secretaría de Acción Femenil de la CNC en el que ella se convirtió en actor y gestor relevante que buscaría el bienestar de los y las campesinas, tanto por razones personales y políticas, que le ayudarían a ascender políticamente de ser una regidora del municipio de San Martín Hidalgo, Jalisco a la Secretaría de Acción Femenil de la CNC federal (1953-1957), y, finalmente, a representar el Décimo Distrito Electoral de Autlán, Jalisco por medio de una diputación federal (1955-1958) ${ }^{20}$ Por tanto, la llegada de Urzúa Flores a la Acción Femenil de la CNC no fue casual, todo un trabajo constante la respaldaba; tuvo que ver con sus capacidades gestoras que venían exhibiéndose desde antes y que se formalizaron en el marco del Congreso de las mujeres priistas en esas mismas fechas de agosto de 1953. Este Congreso marcó la visibilidad de las 120 delegadas, entre ellas Urzúa. La dirigencia nacional del PRI utilizó todo el aparato estatal para exhibir públicamente durante ese congreso la fuerza de la masa femenil a disposición del partido. ${ }^{21}$

$\mathrm{Su}$ ascenso político coincidió con el asesinato de Alfonso Ceballos, cacique de San Martín Hidalgo (1930-1950). El grupo político de Ceballos se debilitó y se abrió el camino para que se consolidara la facción de Urzúa Flores. Su ascenso político se dio en el contexto donde el campo enfrentaba muchos problemas por falta de apoyos crediticios y por una política modernizadora que favorecía la industrialización en vez del fomento agrario. Había un creciente

${ }^{19}$ Ibidem.

${ }^{20}$ CDMGuf-BCCG-CO, 2. Acción Política, 2.1 Acción Femenina-CNC, caja 21, exp. 657, borrador de informe 1957, ff. 17.

${ }^{21}$ Enriqueta Tunón, jPor fin... ya podemos elegir y ser electas! El sufragio femenino en México, 1935-1953 (México: Conaculta, INAH, Plaza Valdés Editores, 2002), 145-149. 
descontento en el sector campesino, dentro y fuera de la CNC, que presionaba por la radicalización de la CNC y por un justo reparto agrario. Los dirigentes de la CNC se enfrentaron con la imagen del agrarismo como "sinónimo de atraco o de asalto". ${ }^{22}$ Éste es el panorama al que Urzúa Flores buscó acercarse con auténticos ejidatarios y campesinos.

La escueta propuesta que le presentó Urzúa Flores a Rodríguez Cano a finales de agosto de 1953, la transformó en un plan de trabajo para el periodo 1953-1956 más detallado y sustentado. Urzúa Flores estipuló en esta propuesta que se debía pugnar por la preparación de más de un millón de mujeres campesinas, sin que perdieran su feminidad. Asimismo, buscaría la dignificación del hogar, la consolidación de la familia, mejorar la vida en los ejidos. Consideraba necesario la participación de las mujeres, pero "sobre bases de igualdad de oportunidades de trabajo, de educación, para el progreso de la democracia y mejor disfrute de la libertad". ${ }^{23}$

Su plan de trabajo lo dividió en tres aspectos: de organización, político y social. La Secretaría de Acción Femenil apoyaría la política agraria y colaboraría en "la elevación económica y moral de los campesinos y el aumento de la producción agrícola", junto con las demás secretarias de la Central. Formaría un Consejo Nacional Femenino y se elaboraría un reglamento. La orientación política de sus afiliadas seguiría los lineamientos del presidente de la República, del PRI y del comité central de la CNC. Se levantaría un censo. La Secretaría de Acción Femenil intervendría en la selección de elementos femeniles nuevos, "auténticos del campo, escogiéndose para ello a las mujeres más capaces que se hayan distinguido en la defensa de los intereses campesinos" ${ }^{24}$ Realizaría reuniones con las dirigentes femeniles de los comités regionales campesinos para conocer sus problemas. Buscaría el acercamiento de las representantes de las li-

${ }^{22}$ Leonardo Lomelí Vanegas, "La consolidación del sistema político mexicano: el periodo de Adolfo Ruiz Cortines”, en El partido de la revolución: institución y conflicto, 1928-1999, Miguel González Compeán y Leonardo Lomelí (México: Fondo de Cultura Económica, 2000), 259-260.

${ }^{23}$ CDMGuf-BCCG-CO, 2. Acción Política, 2.1 Acción Femenina-CNC, caja 19, exp. 597, Plan de trabajo, 1953, ff. 56.

${ }^{24}$ doc. cit. 
gas femeniles estatales con las esposas de los gobernadores y dirigentes del sector popular y obrero, en busca de unidad de acción a favor de las mujeres. Proponía que la Secretaría de Acción Femenil contara con tres organizadoras nacionales femeniles para que la ayudaran en sus trabajos. Elaboraría una cartilla cívica para la mujer campesina y su difusión, así como cartillas sobre la Constitución, el Código Agrario y la Ley del Trabajo. Publicaría un boletín mensual de orientación paras las campesinas. Gestionaría la transmisión de radiodifusiones para las campesinas. Defendería los derechos agrarios de las mujeres. Impulsaría eventos cívicos para conmemorar a héroes y heroínas. Planeaba una reunión nacional de dirigentes femeniles para hacer un diagnóstico de los problemas femeniles. Buscaría fundar escuelas de capacitación en la capital del país, estados y distritos para la formación y orientación de dirigentes femeniles, sujetos a un programa especial. Concebía que las campesinas debían colaborar en las juntas de mejoramiento moral, cívico y material en cada ejido. Asimismo, impulsaría la campańa de alfabetización entre las campesinas para disminuir el índice de mujeres analfabetas. Planeaba luchar contra la carestía de la vida y combatir los monopolios. Aplaudía la iniciativa de la ssa de establecer los Centros-Piloto para la madre y el nińo campesinos, y ayudarían y respaldarían esta política. Proyectaba que la Secretaría de Acción Femenil pidiera a los secretarios de la SSA y de la SEP, se establecieran brigadas móviles de asistencia y alfabetización en regiones remotas.

Urzúa Flores puntualizaba que la Secretaría de Acción Femenil apoyaría en las campańas culturales a favor de la madre y el niño; secundaría la creación de guarderías infantiles; los programas de desayunos escolares; el establecimiento y mejoramiento de maternidades campesinas; fomentaría diversiones sanas; alentaría campañas proconstrucción de escuelas; y gestionaría becas ante la SEP para los hijos de los campesinos en diferentes escuelas.

En el contexto del reconocimiento del sufragio femenino a nivel federal, el 17 de octubre de 1953, Urzúa Flores consideró necesario abordar una cuestión cultural y patriarcal, persuadir a los hombres para que ellos como "los hombres-esposos, hermanos mayores permitieran que la mujer campesina militara efectivamente dentro de 
las filas de la CNC". ${ }^{25}$ Después del reconocimiento del sufragio femenino, el diputado y secretario general de la CNC, Lorenzo Azúa Torres y Urzúa Flores solicitaron, a todas las secretarias de Acción Femenil y a los secretarios generales de las Ligas de Comunidades Agrarias y Sindicatos Campesinos de los estados, que levantaran un censo general de mujeres campesinas de 18 a 21 años para conocer el número de sus afiliadas y las que estaban en edad de votar y de recibir capacitación.

En marzo de 1954, cuatro secretarias de la CNC (general, femenil, educativa y de acción cooperativa) requirieron a todos los secretarios generales de las ligas de comunidades agrarias y de sindicatos campesinos del país que contestaran un cuestionario para realizar un diagnóstico de los problemas específicos, que discutirían del 8 al 10 de mayo en la ciudad de México.

El cuestionario tenía tres secciones de acuerdo con la función de cada secretaria. Respecto a la Secretaría de Acción Femenil, se plantearon quince preguntas sobre cuestiones que se consideraban eran responsabilidad de las mujeres, como el bienestar del hogar y la comunidad en relación con la alimentación, vivienda, salud y cuidado de los niños. Les interesaba conocer con datos cuantitativos de cuántas ligas femeniles funcionaban; en qué lugares; su número de afiliadas, si habían solicitado una parcela para su liga; si recibían servicios médicos coordinados; si había centros materno-infantiles; cuál era la alimentación ordinaria de los campesinos; cómo instruir a la mujer campesina respecto a sus deberes en la política nacional y local; planteaban si las ligas femeniles estarían dispuestas a iniciar una lucha sistemática contra la delincuencia y los vicios y en favor de la salud y las buenas costumbres; se consultaba si las campesinas estarían dispuestas a concurrir a su cabecera municipal para escuchar conferencias sobre derechos civiles, agrarios, obreros y políticos; se preguntaba si había personas capaces y dispuestas a dar dichas conferencias; cómo se podría mejorar la vivienda del campesino; en qué pueblos había mayor urgencia de agua para beber y para uso

${ }^{25}$ CDMGuf-BCCG-CO, 2. Acción Política, 2.1 Acción Femenina-CNC, caja 21, exp. 657, borrador de informe 1957, ff. 17. 
doméstico; se preguntaba si habían hecho solicitudes para remediar tal situación; qué enfermedades eran más frecuentes y cuáles afectaban más a los niños; qué opinaba la Secretaría de Acción Femenil de la Liga sobre el cultivo y aprovechamiento de las parcelas ejidales pertenecientes a mujeres, niños y ancianos; si podrían las campesinas plantar y cuidar almácigos para reforestar los ejidos; qué cría de aves de corral u otros animales domésticos debían incrementarse.

La Secretaría de Acción Juvenil diseñó seis preguntas centradas en los equipos deportivos; cuántos existían; si ellos estaban dispuestos a recibir conferencias sobre deberes cívicos; nociones de derechos constitucional, agrario, obrero, civil e higiene sexual; si podría formar un orfeón de ambos sexos, una orquesta, un grupo teatral u otra agrupación artística; y finalmente, si estarían dispuestos a ir a la ciudad de México 20 jóvenes campesinos, para recibir cursos intensivos sobre veterinaria. Las preguntas de la Secretaría de Acción Cooperativa se enfocaron en obtener información sobre cuántas cooperativas de producción o de consumo existían; qué opinaban sobre su funcionamiento; si se podría organizar una cooperativa de consumo en cada ejido; si estaban dispuestos a escuchar conferencias sobre cooperativismo. ${ }^{26}$

Las preguntas de la Secretaría de Acción Educativa planearon doce interrogantes sobre si las escuelas carecían de maestros; si había inconformidades por el trabajo de los maestros; si se debía aumentar el número de éstos; cómo impulsar la campaña de alfabetización entre campesinos; si se podría aumentar las escuelas nocturnas; si recibían el periódico C.N.C.; si los campesinos estarían dispuestos a pagar por el sostenimiento de este periódico y leer su contenido; si leían otros periódicos o revistas; si había escuelas dirigidas por sacerdotes o monjas; y si había necesidades específicas de las escuelas rurales, de consolidación y escuelas normales rurales. Finalmente, preguntaban si las mujeres tenían acceso a los planteles superiores y

${ }^{26}$ Para una discusión de cooperativismo véase Alicia Civera Cerecedo, "El cooperativismo en la escuela rural del México de los años treinta / The Cooperativism in the Mexican Rural School, 1930s", Anuario de Estudios Americanos 67(2) (julio-diciembre de 2010), 467-491. 
si tendrían acogida cursos de trabajadora social rural en las escuelas de enseñanza agrícola.

Entre abril y mayo de 1953 respondieron los comités ejecutivos de las ligas de comunidades agrarias de Chihuahua, Coahuila e Hidalgo. ${ }^{27}$ En general, reportaron el número de ligas femeniles, constituidas entre 30 y 50 mujeres. Señalaron que la mayoría no recibía servicios médicos, no tenían asignada una parcela, su alimentación se basaba en frijoles, maíz, papa, tortilla y chile y señalaron que también enfrentaban problemas de acceso a agua potable.

El 10 de mayo de 1953, la comisión de estudios integrada por los representantes de las ligas de comunidades agrarias y sindicatos campesinos de Aguascalientes, Durango, Coahuila, Nuevo León, Tamaulipas, Hidalgo y Chiapas acordaron once resoluciones. Éstas se apegaban al plan de trabajo propuesto por Urzúa Flores unos meses antes, pero ahora se especificaron acciones y necesidades más concretas. ${ }^{28}$

La dirigencia de Urzúa Flores en la Secretaría de Acción Femenil en la CNC, seguía tanto los lineamientos del PRI y de la CNC, que ubicaban a las mujeres en el centro de la instrumentación de diversas políticas sociales y las hacía responsables de que se resolvieran problemas educativos, de higiene, nutrición y salud. A diferencia de los líderes revolucionarios burgueses de las décadas de 1920 y 1940 que promovieron una ciudadanía de mujeres restringida bajo el control estatal y patriarcal, ${ }^{29}$ ahora las mujeres debían participar en la política formal y pugnar por el ejercicio de diversos derechos agrarios, civiles, laborales y sociales.

${ }^{27}$ CDMGuf-BCCG-CO, 2. Acción Política, 2.1 Acción Femenina-CNC, caja 19, exp. 608, respuesta de la liga femenil de Chihuahua, 1954, ff. 8; exp. 611, respuesta de la liga femenil de Coahuila, 1954, ff. 5; exp. 612, respuesta de la liga de Hidalgo, 1954, ff. 8.

${ }^{28}$ CDMGuf-BCCG-CO, 2. Acción Política, 2.1 Acción Femenina-CNC, caja 19, exp. 613, informe 1954, ff. 2.

${ }^{29}$ Los objetivos originales que se pretendían conseguir con la fundación de CEIMSA en el periodo cardenista, resultaron tergiversados por las reiteradas acusaciones de corrupción que el henriquismo, particularmente, hizo de esta institución, y del impacto negativo que tuvo esto en la concreción de apoyos para las ligas de mujeres. Véase Heather Fowler-Salamini y Mary Kay Vaughan, "Introducción”, Mujeres del campo mexicano, 1850-1990 (Zamora: El Colegio de Michoacán, Benemérita Universidad Autónoma de Puebla, Instituto de Ciencias Sociales y Humanidades, 2003); Vaughan, "Cultural Approaches to Peasant Politics", 300. 
En una tendencia política que buscaba moldear a las mujeres y permitirles mayor visibilidad, el primer acuerdo estipuló que la CNC, las ligas y los comités regionales campesinos debían tramitar en los gobiernos estatales que se dotara de una parcela ejidal a cada liga femenil. Como cuidadoras del bienestar de la comunidad, también las ligas femeniles junto con la CNC tramitarían ante la SSA que enviara brigadas médicas móviles para atender al campesinado; pero los representantes de los campesinos vigilarían el desempeño de los médicos, no gozarían de autonomía. Este designio entraba en fuerte conflicto con la política de higiene rural de los médicos de la SSA, que presento al inicio de este artículo.

Tal vez uno de los retos más grandes que se les asignó a las ligas femeniles fue que debían mejorar los cultivos agrícolas familiares, con el apoyo de los comités regionales, comisariados ejidales, y negociar con los gobiernos estatales y municipales, mejorar la alimentación del campesinado y su familia. Asimismo, las ligas debían obtener que la Compañía Exportadora e Importadora Sociedad Anónima (CEIMSA) enviara, a los ejidos y comunidades agrarias, alimentos, ropa y calzado a bajo costo. ${ }^{30}$ También deberían gestionar obras de pequeña irrigación, pozos artesianos y la construcción de aljibes familiares. De forma paralela debían fomentar el establecimiento de pequeñas industrias, familiares y caseras. Debían colaborar en las campañas de alfabetización, cívicas, educativas; evitar que funcionaran centros de vicio. Colaborar en las campañas para mejorar la vivienda, combatir las enfermedades y fomentar la reforestación. Se les asignaban muchas acciones y responsabilidades en todos los ámbitos de los ejidos y sus comunidades.

Estas resoluciones asignaban muchas obligaciones a las mujeres. El papel de los secretarios generales de las ligas de comunidades y del

${ }^{30}$ La administración de Lázaro Cárdenas estableció esta Compañía para controlar los precios de ciertos productos agrícolas y alimentos en periodo de escasez de productos y evitar la especulación comercial. Para la década de 1940 también coordinaba una cadena de tiendas subsidiadas por el Estado. Para una discusión más a fondo de CEIMSA véase Enrique Ochoa, "Reappraising State Intervention and Social Policy in Mexico: The Case of Milk in the Distrito Federal during the Twentieth Century", Mexican Studies / Estudios Mexicanos 15(1) (invierno de 1999): 73-99; Aguilar-Rodríguez, "Nutrition and Modernity", 48. 
Estado, a escalas federal, estatal y municipal, no era tan central. Desde la perspectiva de estas resoluciones, los cambios se darían desde abajo por las mujeres de las ligas femeniles. De forma implícita, estas pautas de trasformación se insertaban con políticas públicas construidas desde arriba y desde la ciudad de México, como lo ejemplifican los cursos y sus contenidos. Las acciones que deberían realizar las campesinas, de acuerdo con estas resoluciones, corroboran el argumento de Nichole Sanders de que el PRI no creó el Estado benefactor en México, pero supo capitalizar las influencias internacionales y prescribió cuáles serían las relaciones de género que el movimiento internacional de reformas sociales buscaba: hombres proveedores y jefes de familia y madres al cuidado de sus hijos en el hogar y de su comunidad. Como ha señalado Sanders, la visión estatal y la "científica" en la política social, después de 1940, fue ayudar a los pobres para que aprendieran a auxiliarse a sí mismos. ${ }^{31} \mathrm{~A}$ partir del decenio de 1950, las políticas sociales buscaban la participación de la ciudadanía y de organizaciones caritativas y filantrópicas privadas en la instrumentación de programas sociales para proteger a las familias, en especial, a las madres y los niños pobres, así como la construcción de hospitales, maternidades, comedores familiares, centros infantiles y de maternidad, entre otros. ${ }^{32}$ Así debían hacerlo las campesinas de las ligas femeniles de la CNC. Al final, las acciones de las ligas femeniles cenecistas se tradujeron en la realidad en la mediatización de las propias mujeres.

FormaCiÓN DE NUEVOS CUADROS: CURSOS DE CAPACITACIÓN Y ORIENTACIÓN PARA MUJERES CAMPESINAS

Una de las preocupaciones del Comité Ejecutivo de la CNC en 1954 fue la formación de nuevos cuadros de dirigentes medios en el sector femenil campesino por medio de cursos intensivos de capacitación para mujeres campesinas. El primer curso se llevó a cabo del 1 de mayo al 15 de junio de 1954. Asistieron dos mujeres de cada enti-

${ }^{31}$ Sanders, Gender and Welfare in Mexico.

${ }^{32}$ Ibid. 
dad, quienes debían demostrar que eran auténticas hijas de campesinas sin ningún cargo administrativo. La presidencia de la república y la CNC les pagó el hospedaje y la alimentación, mientras que las ligas de comunidades agrarias de cada estado pagaron los gastos del transporte. ${ }^{33}$

Para la realización de estos cursos, Urzúa Flores se coordinó con el oficial mayor de la SEP, el director general de Alfabetización, el Instituto Nacional de Bellas Artes, la Junta Nacional para el Mejoramiento de la Alimentación del Instituto Nacional de Nutrición y la dirigencia nacional del PRI. Las asistentes recibieron cursos sobre la historia y geografía de México, elementos de economía política, educación cívica, puericultura, higiene general y especial de la boca; cultivos, fruticultura, hortalizas y cría de animales; labores domésticas, costura, cocina, conservación de frutas y carnes, quesos, mantequilla; elementos de derecho agrario e industrial; campaña de alfabetización, turismo; deportes y educación física.

Las inauguraciones y las clausuras siguieron el protocolo de cualquier ceremonia cívica y educativa, el objetivo era inspirar disciplina y orden en un marco institucional y nacionalista. En el informe de la gestión de Urzúa Flores como secretaria de Acción Femenil, afirmaba que los resultados que se obtuvieron fueron de un alcance insospechado porque logró formar nuevos cuadros en la provincia. Sostenía que

gracias a estas nuevas dirigentes, la $\mathrm{CNC}$, tiene a sus mejores abanderadas y portavoces, quienes organizan a sus compañeras, editan periódicos rurales, fundan costureros, hacen juguetería para los niños, [ilegible] regionales, mejoran las artesanías, conservan frutas y legumbres, promueven distracciones sanas entre el pueblo, difunden lo mejor de nuestro folklor nacional, inician la apertura de nuevas escuelas, realizan labores de alfabetización, intervienen en las campańas sociales (esto es muy importante)

${ }^{33}$ CDMGuf-BCCG-CO, 2. Acción Política, 2.1 Acción Femenina-CNC, caja 19, exp. 593, cursos 1954, ff. 2; exp. 602, bases de los cursos, 1954, ff. 2, exp. 627, cursos, 1954, ff. 2; caja 20, exp. 629, varias invitaciones a la inauguración y clausura de cursos, 19541965, ff. 29; exp. 643, cursos, 1956, ff. 1. 
tienen ya una conciencia de clase campesina, muy sincera política de su cometido y una actitud de trabajo para engrandecer a México. ${ }^{34}$

Bajo la dirigencia de Urzúa Flores se coordinaron otros dos entre 1955 y 1956 . En la década de 1960, estuvo a cargo de organizarlos. Así, la CNC daba continuidad y seguimiento a la formación de los nuevos cuadros de diversas comunidades.

\section{LAS ELECCIONES Y ORIENTACIÓN POLÍTICA EN I 955}

A finales de 1954 y principios de 1955, Urzúa Flores intensificó su campańa de educación cívica y política para preparar a las campesinas para las elecciones de julio de 1955. Como dirigente de la CNC, les sugirió a las secretarias de acción femenil de las Ligas de Comunidades Agrarias y a las secretarias generales de las ligas femeniles campesinas que debían convencer a las mujeres mexicanas de 18 ańos si eran casadas o solteras de 21 ańos, que se registraran en las oficinas del padrón electoral y obtuvieran su credencial de elector. También las secretarias de la sección femenil y las ligas femeniles debían promover que las mujeres casadas se empadronaran con el apellido de su marido, para que el día de elección votaran acompanaadas de él y sus hijos, como menores incapacitadas para ejercer su ciudadanía. ${ }^{35}$

De forma simultánea, Urzúa Flores emprendió su campaña para la diputación federal del x Distrito de Autlán, Jalisco; continuó atendiendo las diversas peticiones y gestiones que llegaban a la Secretaría de Acción Femenil. En los primeros siete meses de 1955, Urzúa Flores enfrentó muchas resistencias por la incorporación de las mujeres en la representación formal partidista. Para intentar detener la presencia de las mujeres en la política formal, los hombres recurrieron a difundir rumores para desprestigiar la fama y la

${ }^{34}$ CDMGuf-BCCG-CO, 2. Acción Política, 2.1 Acción Femenina-CNC, caja 21, exp. 657, borrador de informe 1957, ff. 17.

${ }^{35}$ CDMGuf-BCCG-CO, 2. Acción Política, 2.1 Acción Femenina-cnc, caja 20, exp. 631, circular sobre las elecciones, 1955, ff. 10. 
legitimidad de Urzúa Flores; su vida personal y amorosa se sometió a una crítica en la opinión pública. ${ }^{36} \mathrm{Al}$ final, ella ganó las elecciones con 22,476 votos contra 5,471 votos para el Pan. ${ }^{37}$ Fue impugnada por un diputado panista, pero la Cámara de Diputados la ratificó.

Después de las elecciones de julio, el comité central de la CNC solicitó a María de Jesús Medina, secretaria de Acción Femenil de la Liga de Comunidades Agrarias de Guadalajara, que recabara información sobre el proceso electoral para conocer de forma detallada los errores observados y los aciertos en relación con la participación de las mujeres en esta contienda electoral. ${ }^{38}$ Aunque en términos generales, oficialmente, la CNC y la prensa nacional y de Guadalajara reportaron que "el sufragio popular fue ejercitado de manera ejemplar y pacíficamente por las mujeres", había mucho descontento de mujeres PRIistas por los malos tratos, discriminación y porque aún se les consideraba como ineptas en la política. ${ }^{39}$

Como diputada federal y como secretaria de Acción Femenil no sólo se enfocó a atender y formar a campesinas, sino también veló por asuntos muy diversos sobre problemas ejidales entre campesinos, despojos; conflictos ganaderos; construcción de caminos vecinales, presas, obras de riego e introducción de agua potable; realizó gestiones para la construcción de escuelas, nombramiento de profesores para escuelas; solicitó material escolar y becas para campesinos para realizar sus estudios en la Escuela Normal Rural de San Marcos, Zacatecas; negoció los nombramientos de la ssa de los promotores sociales para que instalaran Centros de Bienestar Social Rural; informó sobre la emigración de campesinos como braceros; realizó

${ }^{36}$ CDMGuf/BCCG, CO, 2. Acción Política, 2.14 PRI, 2.14.3 Procesos y campañas de candidatos a senadurías, diputaciones, año [1955], caja 137, exp. 4944, ff 1, 2 y exp., 4929, ff 1 ,

${ }^{37}$ ACU, Diario de Debates de la H. Cámara de Diputados, Legislatura XlıII, Año Legislativo I, periodo ordinario, Diario núm. 6, 24 de agosto de 1955.

${ }^{38}$ CDMGUf-BCCG-CO, 2. Acción Política, 2.1 Acción Femenina-CNC, caja 20, exp. 636, circular sobre las elecciones, 1955, ff. 2.

${ }^{39}$ CDMguf-BCCG-CO, 2. Acción Política, 2.1 Acción Femenina-CNC, caja 23, exp. 687, Dirigentes de Chihuahua exponen la falta de preparación cívica y la exclusión en asuntos políticos, 1959, ff. 4 . 
solicitudes para que en el programa de radio la "Hora Nacional" lo dedicaran a algún municipio. Asimismo, pidió mobiliario e instrumental para diversos hospitales civiles rurales.

\section{NUTRICIÓN Y CONSUMO}

Además de la campaña de alfabetización, cívica y política, Urzúa Flores se interesó mucho en las políticas y programas para mejorar la calidad de alimentos que consumía la población en general. Como las trabajadoras sociales, reformadoras sociales y voluntarias de clase media de la Ciudad de México que trabajaban para la SsA en puestos intermedios en conjunto con los hombres en puestos directivos, que cabildearon y gestionaron con éxito programas sociales en favor de las madres pobres y de sus niños, ${ }^{40}$ Urzúa Flores hizo algo similar desde la CNC, pero su objetivo era llegar a las mujeres en zonas rurales. Invitó a los cursos al Dr. Jesús Díaz Barriga, médico nicolaita, cardenista, que entre 1928 y 1932 había propuesto una reforma radical de higiene rural y servicios médicos rurales, en el decenio de 1950 dirigía la Junta Nacional para el Mejoramiento de la Alimentación del Instituto Nacional de Nutrición (INN). ${ }^{41}$ El proyecto de política de higiene rural del Dr. Díaz Barriga de la década de 1920 también incluía cambiar la dieta de la población en general para mejorar la calidad de vida, de trabajo y disminuir las enfermedades que aquejaban a la mayoría de la población. La historiadora Ana María Kapelusz-Poppi señala que el Dr. Barriga tuvo gran interés por crear recetas con alto valor nutritivo y le pidió a su esposa que elaborara algunas en este sentido. ${ }^{42}$

${ }^{40}$ Sanders, Gender and Welfare in Mexico.

${ }^{41}$ Kapelusz-Poppi, "Rural Health and State Construction", 261; "Physician Activists and the Development of Rural Health", 38-39. Para un excelente estudio sobre la comida y la construcción de la identidad mexicana véase Jeffrey M. Pilcher, ;Vivan los tamales! La comida y la construcción de la identidad mexicana (México: Ciesas, Conaculta, 2001).

42 Ana María Kapelusz-Poppi, "Provincial Intellectuals from Michoacan and the Professionalization of the Post-revolutionary Mexican State" (Tesis de doctorado, University of Illinois, 2002), 240; "Physician Activists and the Development of Rural Health", 39. 
En el decenio de 1950, el Dr. Barriga, el Dr. José Quintín Olascoaga Moncada, considerado el dietista pionero en México, ${ }^{43}$ y la Dra. Juana Navarro García de Meyer, la primera médica egresada de la Universidad de Guadalajara en 1930, ${ }^{44}$ fundadora de la Alianza de Profesoras de Obstetricia y Enfermeras de Guadalajara en 1935, investigaron acerca de las cuestiones de nutrición en México. ${ }^{45}$

De acuerdo con Gabriela Castańeda López y Ana Cecilia Rodríguez, la Dra. Navarro fue instructora de visitadoras rurales del Departamento de Salubridad de Jalisco. De 1940 a 1942, ella y "los doctores José Quintín Olascoaga, Rafael Ramos Galván y Guadalupe Eguiluz, estudiaron en el Instituto Nacional de Nutrición de Buenos Aires". 46 La Dra. Navarro obtuvo el grado de dietista y médico dietólogo. $\mathrm{Al}$ regresar se incorporó al recién creado INN en 1942, junto con el Dr. Olascoaga ${ }^{47}$ En 1955, el Dr. Jesús Díaz Barriga y la Dra. Juana Navarro presentaron la ponencia "La nutrición y la productividad del trabajo en el medio rural" en el Sexto Congreso de Sociología, celebrado en Morelia. ${ }^{48}$

En los Cursos de Capacitación y Orientación para Mujeres Campesinas que organizó Urzúa Flores en la Ciudad de México entre 1954 y 1957, las campesinas recibieron diversas clases, entre

${ }^{43}$ Aguilar-Rodríguez, "Milk-Consumption”, 41.

${ }^{44}$ María Teresa Fernández Aceves, "Las mujeres graduadas en la Universidad de Guadalajara, 1925-1933”, en Historia Social de la Universidad de Guadalajara, coord. Carmen Castañeda, 113 (Guadalajara: Universidad de Guadalajara, Ciesas, 1995).

${ }^{45}$ Archivo Histórico de Jalisco, Ramo de Trabajo, T-9-934, caja 3. En el I Congreso de Higiene Rural, organizado por el Dr. Barriga y el Dr. Arreguín Vélez en 1935 en Morelia, la Alianza de Profesoras de Obstetricia y Enfermeras del Estado de Jalisco defendió el trabajo de las parteras ante los médicos. Véase Kapelusz-Poppi, "Provincial Intellectuals from Michoacán"; María Teresa Fernández Aceves, "Debates sobre el ingreso de las mujeres a la universidad y las primeras graduadas en la Universidad de Guadalajara, 1914-1933", La Ventana (21) (julio de 2005): 100-101.

${ }^{46}$ Castañeda López y Rodríguez de Romo, Desafiando a la tradición, 75-79.

${ }^{47}$ Ibid.

48 "Crónica del Sexto Congreso Nacional de Sociología", Revista Mexicana de Sociología (18)(1) (1956): 225-230. Las ponencias del Sexto Congreso Nacional de Sociología se publicaron en 1962, en éste se incluyó la ponencia que presentaron Jesús Díaz Barriga y Juana Navarro G. de Meyer, con el título "La Nutrición y la productividad del trabajo en el medio rural”, véase "Back Matter”, Revista Mexicana de Sociología, 24(3), (1962): 979. 
ellas, cocina y nutrición. En estos cursos la CNC reprodujo en copias mimeografiadas, con autorización de la Dra. Juana Navarro (también de la Junta Nacional para el Mejoramiento de la Alimentación del INN), recetarios elaborados por la Dra. Navarro y las amas de casa María Inés Cardona de Díaz Barriga y Gloria Pardo de Díaz Barriga.

Los recetarios de la Dra. Navarro y el ama de casa Cardona de Díaz Barriga, los habían preparado con la colaboración del INN y de CEIMSA. Las recetas incluían cuatro grupos de productos con alto valor nutritivo: pescados secos, leche descremada en polvo, soya y garbanzo. En algunas de éstas se recomendaba incorporar el consumo de la leche en polvo descremada porque tenía "las ventajas de ser económica, de que puede aumentar el consumo, especialmente entre los niños, quienes se nutri[rían] mejor y forma[rían] el hábito de tomarla: la generalización de éste [sic] ábito [era] la base para el desarrollo futuro de la industria lechera nacional". ${ }^{49}$ Además del alto valor nutritivo del pescado seco, la soya y el garbanzo y la leche descremada en polvo, se promovía su consumo porque eran muy baratos. A lo largo de los recetarios se explicaban sus ventajas de consumo, su valor nutritivo y porque eran muy económicos. Así lo argumentaron la Dra. Navarro y la Sra. Cardona de Díaz Barriga, "50 gramos de proteína de soya cuestan cuando mucho 30 centavos, mientras que los mismos 50 gramos de proteínas de carne o de leche fresca cuestan $\$ 1.60$ aproximadamente o de huevo $\$ 2.75$; los 50 gms.; de proteína descremada en polvo valen unos 80 centavos". ${ }^{50}$

Muy probablemente desde este contacto con los doctores Díaz Barriga y Navarro, y al familiarizarse con sus investigaciones, programas y políticas, motivaron a Urzúa Flores a recopilar recetas culinarias de todos los municipios, comunidades y rancherías que visitaba. Ella concordaba con el objetivo de los programas del INN, de que, al mejorar la nutrición del campesinado, disminuirían las enfermedades y aumentaría la productividad. ${ }^{51}$ Aquí podría compa-

${ }^{49}$ CDMGuf-BCCG-CO, 2. Acción Política, 2.2.1 Bienestar Social, caja 30, exp. 883, recetas de cocina para alimentos más nutritivos, 1953-1955, ff. 34.

${ }^{50}$ doc. cit.

${ }^{51}$ En la CDMGUF-BCCG-CO hay gran cantidad de documentos sobre las necesidades 
rar la frase clásica de la feminista estadounidense Joan Scott, "cómo la política construye al género y cómo el género construye la política"; 52 en el caso de Guadalupe, sería "cómo la política cocinó la comida y como al preparar la comida, se cocinó la política”.

\section{IMPACTO Y RESULTADOS DE LA POLÍTICA} de URZÚa Flores EN LA CNC

¿Cuál fue el impacto de los Cursos de Capacitación y Orientación para Mujeres Campesinas y de programas de nutrición que impulsó Urzúa Flores dentro de las ligas femeniles? Un informe que envió Guadalupe Martínez, secretaria de Acción Femenil de Coahuila a Urzúa Flores brinda pistas interesantes. Martínez detallaba las diversas labores que realizaba la Liga Femenil en Torreón, desde actividades cívicas como el desfile del 20 de noviembre; la jornada cívica de la Revolución; o la inauguración de una noria de agua potable; y las utilidades que obtenían de la parcela de la Liga Femenil. Respecto a "la Pasteurizadora Laguna" estaba constituida por una sociedad con acciones muy caras, por lo que la gente de pocos recursos no puede participar. La leche de esta sociedad sólo se vendía en expendios en la ciudad de Torreón y aún no llegaba a los ejidos.

A pesar de que los doctores Díaz Barriga, Olascoaga Moncada y Navarro impulsaron desde el InN y la Junta Nacional para el Mejoramiento de la Alimentación, el consumo de la leche para ayudar a consolidar una industria nacional moderna, fue muy difícil llevarlo a cabo como lo informó esta líder campesina y como también lo ha mostrado la investigación de la historiadora Sandra Aguilar-Rodríguez sobre el consumo de la leche en la Ciudad de México. ${ }^{53}$

Sobre los servicios médicos ejidales, Martínez señaló que colaboraban médicos muy bien remunerados, pero el problema era la escasez de medicamentos. Sobre el Programa de Bienestar Social,

de los diferentes municipios que Urzúa Flores representó en la Cámara de Diputados, y al reverso ella anotó un sinnúmero de recetas.

${ }^{52}$ Joan Wallach Scott, Gender and the Politics of History (Nueva York: Columbia University Press, 1988): 44-50.

${ }^{53}$ Aguilar-Rodríguez, "Nutrition and Modernity". 
consideraba que lo único que se había aprovechado era la campaña de inmunización en los ejidos y los cursos de corte y confección. Terminaba su informe "el aspecto de nutriología no ha tenido gran estímulo entre las gentes del campo, no por culpa del personal que lo atiende, sino por el escaso medio con que cuentan las mismas gentes del campo". ${ }^{54}$

Respecto a la capacitación política, las secretarias femeniles de los tres sectores del PRI en Chihuahua en 1959 se quejaron amargamente porque las trataban muy mal en el partido y porque aún las consideraban no capaces de participar activamente en la contienda electoral presidencial de $1958 .^{55}$ A pesar de que Urzúa Flores realizó una intensa labor de orientación cívica por medio de reuniones, ceremonias cívicas, circulares, documentos instructivos y de la edición de un periódico, Alborada Femenil (1954-1957), ${ }^{56}$ y la publicación de Ud. ya es ciudadana, Decálogo para nuestras "voluntarias" del Ejército Femenino Protector de la Infancia y de la Salud y El ideario de la mujer campesina para despertar interés por los problemas de mujeres, aún había un abismo entre programas, políticas y prácticas. Pero definitivamente, Urzúa Flores sí logró gestionar diferentes obras públicas que beneficiaban a la población en general como la construcción de hospitales, escuelas, pozos de agua y caminos.

\section{Consideraciones finales}

Los programas, resoluciones y acciones que debían emprender las ligas femeniles de la CNC se enmarcaban con lo que Mary Kay Vaughan llama la modernización del patriarcado durante el periodo posrevolucionario. Las mujeres colaborarían junto con el Estado en acciones modernizadoras en la educación, la salud, nutrición y la

${ }^{54}$ CDMGuf-BCCG-Co, 2. Acción Política, 2.1 Acción Femenina-CNC, caja 20, exp. 648, informe de la Liga Femenil de Torreón, 1956, ff. 2.

${ }^{55}$ CDMGuf-BCCG-CO, 2. Acción Política, 2.1 Acción Femenina-CNC, caja 23, exp. 687, Dirigentes de Chihuahua exponen la falta de preparación cívica y la exclusión en asuntos políticos, 1959, ff. 4 .

${ }^{56}$ De acuerdo con el informe de Urzúa Flores de 1957, se distribuyeron 7,500 por número en todo el país. 
protección de los niños, en concreto, en el bienestar de su comunidad y de su hogar. Con esta movilización se pensó que las mujeres se empoderarían, pero en la década de 1950 eran las mujeres de clase media y alta quienes podían ejercer más sus derechos civiles, laborales y políticos, que las campesinas y trabajadoras. ${ }^{57} \mathrm{Las}$ campesinas aún tenían muy poco acceso a distintos recursos para su empoderamiento a través de derechos agrarios y laborales. Por una parte, el Estado, hacedores de políticas públicas y líderes políticos percibieron a las mujeres como responsables del bienestar de la comunidad. Ellas debían aprender a auxiliarse y resolver los problemas de su comunidad. Por otra parte, las catorce representantes de las ligas femeniles de la Laguna que demandaban garantías "para formar un hogar modesto", recurrían a lo que Temma Kaplan denomina los derechos de género centrados en preocupaciones sociales y de sobrevivencia. ${ }^{58}$ Ambas visiones, la del Estado y las mujeres, convergieron en el programa que presentó Urzúa Flores, en las resoluciones que elaboró la comisión de estudios de la Secretaría de Acción Femenil de la CNC y en las acciones y reacciones de las secretarias femeniles de la CNC.

Aún falta reconstruir más experiencias locales de los programas, políticas sociales y acciones para conocer cómo funcionó el Estado, el PRI y sus organizaciones corporativas en diferentes escalas. Con estos elementos se podrá evaluar qué intentó llevar a cabo Urzúa Flores junto con otras agencias gubernamentales. Este rescate histórico nos permitirá valorar de forma más matizada cómo se diseñaron las políticas públicas, qué actores participaron en su planeación y en su instrumentación y cómo se resistieron y negociaron.

${ }^{57}$ Véase Heather Fowler-Salamini y Mary Kay Vaughan, "Introducción”, Mujeres del campo mexicano, 1850-1990 (Zamora: El Colegio de Michoacán, Benemérita Universidad Autónoma de Puebla, Instituto de Ciencias Sociales y Humanidades, 2003); Vaughan, "Cultural Approaches to Peasant Politics", 300.

${ }^{58}$ Para Kaplan, "aquéllas con conciencia femenina aceptaban el sistema de género de la sociedad que asigna a la mujer la responsabilidad de preservar la vida". Temma Kaplan, "Female Consciousness and Collective Action: The Case of Barcelona, 1910-1918", Signs 7(3) (primavera de 1982): 545. 
Bibliografía

Aguilar-Rodríguez, Sandra. "Cooking Modernity: Nutrition Policies, Class, and Gender in 1940s and 1950s Mexico City". The Americas 64(2) (octubre 2007): 177-205.

Blum, Ann S. Domestic Economies: Family, Work, and Welfare in Mexico City, 1884-1943. Lincoln: University Nebraska Press, 2009.

Castañeda López, Gabriela y Ana Cecilia Rodríguez de Romo. Desafiando a la tradición: las primeras egresadas de las escuelas de medicina de México, 1887-1936. México: Academia Nacional de Medicina de México, 2014.

Civera Cerecedo, Alicia. "El cooperativismo en la escuela rural del México de los años treinta / The Cooperativism in the Mexican Rural School, 1930s". Anuario de Estudios Americanos 67(2) (julio-diciembre de 2010): 467-491.

Fernández Aceves, María Teresa. "Las mujeres graduadas en la Universidad de Guadalajara, 1925-1933”. En Historia Social de la Universidad de Guadalajara, coord. Carmen Castañeda, $97-$ 122. Guadalajara: Universidad de Guadalajara, Ciesas, 1995.

"Debates sobre el ingreso de las mujeres a la universidad y las primeras graduadas en la Universidad de Guadalajara, 19141933”. La Ventana (21) (julio de 2005): 90-106.

" "Las políticas de género de la Confederación Nacional Campesina y el liderazgo de María Guadalupe Urzúa Flores, 1950-1960". En Mexico in Transition: New Persectives on Mexican Agrarian History, Nineteenth and Twentieth Centuries / México y sus transiciones: reconsideraciones sobre la historia agraria mexicana, siglos XIX y XX, ed. Antonio Escobar Ohmstede y Mattew Butler, 565-600. México: Ciesas, Universidad de Texas-Austin, 2013.

Mujeres en el cambio social en el siglo XX mexicano. México: Siglo XXI Editores, Ciesas, 2014.

Fowler-Salamini, Heather y Mary Kay Vaughan. "Introducción”. Mujeres del campo mexicano, 1850-1990. Zamora: El Colegio de Michoacán, Benemérita Universidad Autónoma de 
Puebla, Instituto de Ciencias Sociales y Humanidades, 2003, 27-46.

HewitT de Alcántara, Cynthia. La modernización de la agricultura mexicana, 1940-1970. México: Siglo XXI Editores, 1988.

López, Oresta. Alfabeto y enseñanzas domésticas. El arte de ser maestra rural en el Valle del Mezquital. México: Ciesas, Consejo Estatal para la Cultura y las Artes de Hidalgo, 2001.

Kapelusz-Poppi, Ana María. "Physician Activists and the Development of Rural Health in Postrevolutionary Mexico". Radical History Review (80) (primavera 2001): 35-50.

. "Rural Health and Status Construcción in Post-Revolucionar Mexico: The Nicolita Project Foro Rural Medical Services". The Américas 58(2) (octubre 2001): 261-283.

"Provincial Intellectuals from Michoacan and the Professionalization of the Post-revolutionary Mexican State". Tesis de Doctorado, University of Illinois, 2002.

Kaplan, Temma. "Female Consciousness and Collective Action: The Case of Barcelona, 1910- 1918”. Signs 7(3) (primavera de 1982): 545-566.

Lomelí Vanegas, Leonardo. "La consolidación del sistema político mexicano: el periodo de Adolfo Ruiz Cortines". En El partido de la revolución: institución y conflicto, 1928-1999. Miguel González Compeán y Leonardo Lomelí, 253-284. México: Fondo de Cultura Económica, 2000.

Mitchell, Stephanie y Patience A. Schell, eds. The Women's Revolution, Mexico (1910-1953). Nueva York: Roman and Littlefield, 2006.

Molyneux, Maxine. "Prefacio". En Los códigos del género. Prácticas del derecho en el México contemporáneo, ed. Helga Baitenmann, Victoria Chenaut y Ann Varley, 7-15. México: unAM-PUEG, Fondo de Desarrollo de las Naciones Unidas para la Mujer, UNIFEM, 2010.

OсноA, Enrique. "Reappraising State Intervention and Social Policy in Mexico: The Case of Milk in the Distrito Federal during the Twentieth Century". Mexican Studies / Estudios Mexicanos 15(1) (invierno de 1999): 73-99. 
Olcott, Jocelyn. Revolutionary Women in Postrevolutionary Mexico. Durham: Duke University Press, 2005.

"'Take off that streetwalker's dress': Concha Michel and the Cultural Politics of Gender in Postrevolutionary Mexico", Journal of Women's History 21(3) (otoño de 2009): 36-59.

Peniche Rivero, Piedad. El movimiento feminista de Elvia Carrillo Puerto y las igualadas: un liderazgo cultural en Yucatán”. En Dos mujeres fuera de serie: Elvia Carillo Puerto y Felipa Poot, ed. Piedad Peniche Rivero y Kathleen R. Martín, 15-69. Mérida: Instituto de la Cultura de Yucatán, 2007.

Pilcher, Jeffrey M. ;Vivan los tamales! La comida y la construcción de la identidad mexicana. México: Ciesas, Conaculta, 2001.

PorTEr, Susie S. Mujeres y trabajo en la ciudad de México: condiciones materiales y discursos públicos (1879-1931). Zamora: El Colegio de Michoacán, 2008.

RockWell, Elsie y Eugenia Roldán Vera. "State Governance and Civil Society in Education: Revisiting the Relationship". En Paedagogica Historica 49(1) (2013): 1-16.

SAnders, Nichole. Gender and Welfare in Mexico: the Consolidation of a Postrevolutionary State. University Park: Pennsylvania State University Press, 2011.

Schell, Patience A. Church and State Education in Revolutionary Mexico City. Tucson: University of Arizona Press, 2003.

Smith, Stephanie J. Gender and the Mexican Revolution: Yucatan Women and the Realities of Patriarchy. Chapel Hill: University of North Carolina Press, 2009.

Scoтt, Joan Wallach. Gender and the Politics of History. Nueva York: Columbia University Press, 1988.

Tuñón, Enriqueta. jPor fin... ya podemos elegir y ser electas! El sufragio femenino en México, 1935-1953. México: Conaculta, INAH, Plaza Valdés Editores, 2002.

Vaughan, Mary Kay. "Cultural Approaches to Peasant Politics in the Mexican Revolution". The Hispanic American Historical Review 79(2) (mayo 1999): 269-305.

. "Modernizing Patriarchy: State Policies, Rural Households, and Women in Mexico, 1930-1940". En Hidden Histories of 
Gender and State in Latin America, ed. Maxine Molyneux y Elizabeth Dore, 194-214. Durham: Duke University Press, 2000. - La política cultural de la revolución. México: Fondo de Cultura Económica, 2001.

Zaremberg, Gisela. Mujeres, votos y asistencia social en el México priista y la Argentina peronista. México: flacso México, 2009. 